\title{
Ist unerfüllter Kinderwunsch ein Leiden? - Der Leidensbegriff im Kontext der Kinderwunschtherapie
}

\author{
Anna Maria Westermann • Ibrahim Alkatout $\mathbb{D}$
}

Eingegangen: 19. Juni 2019 / Angenommen: 12. November 2019 / Online publiziert: 5. Dezember 2019 (C) Der/die Autor(en) 2019

Zusammenfassung Der Begriff Leiden ist in der Medizin und in der Bioethik bisher kaum reflektiert und dahingehend in normativer Hinsicht wenig bestimmt. Dennoch bildet das Leiden an einer Unfruchtbarkeit den Ausgangspunkt für die medizintechnischen Interventionen der assistierten reproduktionsmedizinischen Behandlung. Dabei wird implizit angenommen, dass der unerfüllte Kinderwunsch ein Leiden ist. Ob der unerfüllte Kinderwunsch allerdings ein Leiden darstellt, ist bisher nicht eindeutig geklärt worden.

Ziel dieses Beitrages ist es, die Annahme, dass es sich beim unerfüllten Kinderwunsch um ein Leiden handelt, zu überprüfen. Anhand der Darstellung einiger gängiger Leidenskonzeptionen werden Merkmale von Leiden herausgearbeitet, die als treffende Grundannahmen für eine Leidensbestimmung gelten können. Es wird sich zeigen, dass der unerfüllte Kinderwunsch, entsprechend der Leidenskonzeptionen, als ein Leiden angesehen werden sollte, und ihm somit ein normativer Stellenwert zukommt. In einem weiteren Schritt ist zu klären, ob das Leiden an einem unerfüllten Kinderwunsch als ein Rechtfertigungsgrund für reproduktionsmedizinische Interventionen gelten kann. Dafür wird zum einen der Stellenwert von Leiden, als eine anthropologische Grundbedingung, im Zusammenhang mit dem Leidenslinderungsauftrag der Medizin diskutiert. Zum anderen werden die Risiken der reproduktionsmedizinischen Therapien sowie deren Bedeutung als Gesundheitsressourcen erörtert. Dabei wird deutlich, dass Leiden an einem unerfüllten Kinderwunsch immer ein psychosomatischer Komplex ist. Nur unter Berücksichtigung der

Dr. A. M. Westermann, M.D., B.A.

Abteilung für Gynäkologie und Geburtshilfe, Städtisches Krankenhaus Kiel, Kiel, Deutschland

Prof. Dr. I. Alkatout, M.D., PhD, M.A., MaHM ( $\varangle)$

Kiel School of Gynaecological Endoscopy, Klinik für Gynäkologie und Geburtshilfe, University Hospitals Schleswig-Holstein, Campus Kiel, Arnold-Heller-Str. 3, House 24, 24105 Kiel,

Deutschland

E-Mail: kiel.school@uksh.de 
psychoexistenziellen Dimension des Leidens ergibt sich eine Legitimation für eine angemessene somatische Intervention.

Schlüsselwörter Leiden · Unerfüllter Kinderwunsch · Leidenslinderung · Kinderwunschbehandlung $\cdot$ Reproduktionsmedizin

\title{
Is the unfulfilled desire to have children a form of suffering?-Suffering in the context of reproductive medicine
}

\begin{abstract}
Definition of the problem In medicine and bioethics, the term "suffering" is not clearly defined from a normative point of view. Nevertheless, suffering due to infertility is the starting point for medical interventions in assisted reproductive medicine. This implies that the unfulfilled desire to have children is a form of suffering, but the validity of this statement has not yet been clarified.

Arguments Based on descriptions of some common concepts, certain characteristics of suffering are identified. We discuss the significance of suffering as an anthropological condition in connection with the mission of medicine to alleviate human suffering. Furthermore, the risks of reproductive treatment and their significance for health are addressed.

Conclusion We conclude that the unfulfilled desire to have children is a form of suffering, and therefore has a normative value. The legitimacy of appropriate somatic intervention can only be established by taking the psycho-existential dimension of suffering into account.
\end{abstract}

Keywords Suffering $\cdot$ Infertility $\cdot$ Unfulfilled desire to have children $\cdot$ Relief of suffering $\cdot$ Reproductive medicine

\section{Einführung}

Die Linderung von Leiden ist eine wesentliche Aufgabe der Medizin und ein stetig verfolgtes Ziel (Callahan 1999). Der Leidensbegriff wird auf Grund seines normativen Charakters und der damit verbundenen appellativen Kraft im medizinethischen Kontext zunehmend als moralisches Argument beansprucht. Leiden wird dabei als Grund zur Legitimierung ethisch umstrittener ärztlicher Behandlungen angeführt, wie am Beispiel der Leidenslinderung in der Sterbebegleitung gezeigt wurde (Bozzaro 2015b, 2015a). Allerdings wird eine Auseinandersetzung mit dem Leidensbegriff an sich und eine Reflexion desselben sowohl in der Medizin als auch in der Bioethik vernachlässigt (Bozzaro 2015b; Callahan 2014; Green und Palpant 2014). Dies gilt auch für den Bereich der assistierten reproduktionsmedizinischen Behandlung (ART) (Lauritzen 2014). Ein unerfüllter Kinderwunsch wird im allgemeinen Sprachgebrauch sowie im klinischen Alltag als ein Leiden an einer Unfruchtbarkeit bezeichnet (Strauß et al. 2004). Ob und inwiefern der unerfüllte Kinderwunsch aber als ein Leiden angesehen werden kann und welche Implikationen sich für den Umgang mit der ART daraus ergeben, ist wissenschaftlich bislang wenig aufgearbeitet 
worden. Darauf ist zuletzt in der Diskussion um die Rechtfertigung von Uterustransplantationen hingewiesen worden. Das Leiden an einem unerfüllten Kinderwunsch stellt den Ausgangspunkt für diverse reproduktive Techniken, einschließlich der Uterustransplantation als dem komplexesten Fall der künstlichen Fortpflanzung, dar. Deshalb ist sein normativer Status klärungsbedürftig (Bozzaro et al. 2019).

In der neuen Richtlinie ,Zur Entnahme und Übertragung von menschlichen Keimzellen im Rahmen der assistierten Reproduktion“" ist eine Systematisierung von verbindlichen Regelungen zur assistierten Reproduktion hinsichtlich der medizintechnischen Interventionen erfolgt (Montgomery et al. 2018). Der unerfüllte Kinderwunsch wird dabei explizit als der Grund für die Behandlungsmaßnahmen der Reproduktionsmedizin benannt. Demgegenüber ist aber nicht nur das Verhältnis zwischen unerfülltem Kinderwunsch und dem Leidensbegriff unbestimmt, sondern auch sein Status als Krankheit ist umstritten. Ob und unter welchen Bedingungen eine Sterilität als Krankheit angesehen wird, ist Gegenstand gegenwärtiger ethischer und rechtlicher Diskussionen, die unter anderem auch für die Kostenerstattung der Behandlungen durch die Krankenkassen relevant sind (Krones et al. 2006; Wehmeyer 2012). Es ist umstritten, ob die reproduktionsmedizinischen Techniken im herkömmlichen Sinne ein Heilmittel darstellen oder vielmehr als Mittel zur Erfüllung eines reinen Wunsches anzusehen sind. Deshalb ist die Grenze zwischen der Reproduktionsmedizin und der sogenannten ,Wunscherfüllenden Medizin“ unscharf (Krones et al. 2006; Buyx und Hucklenbroich 2009; Kettner 2006, 2009).

Unter diesen Gesichtspunkten ist die moralische Bedeutsamkeit des Leidensbegriffes als Rechtfertigung für die möglicherweise risikoreichen Therapien der assistierten Reproduktion ungeklärt. Der Leidensbegriff bedarf in Bezug auf die Kinderwunschtherapie einer umfassenderen Bestimmung, um seinen normativen Stellenwert festzulegen. Im Folgenden soll deshalb der Begriff Leiden ethisch reflektiert und seine Verwendung in Bezug auf den unerfüllten Kinderwunsch diskutiert werden.

\section{Der Stellenwert des Leidens als eine „conditio humana“ in der Medizin}

Leiden ist eine „conditio humana“. Es ist eine existenzielle menschliche Selbstund Welterfahrung in dem Sinne, dass in dieser Erfahrung die eigene Begrenztheit wahrgenommen wird. Krankheit und Leiden stellen - wie auch das Wissen um den eigenen Tod - Kontingenzerfahrungen dar, die untrennbar mit dem Menschsein verbunden sind. Das Aufheben dieser Erfahrungen stellt somit das Menschsein selbst in Frage (Jaspers 1973). Leiden als ein multidimensionales Geschehen ist deshalb untrennbar mit der Frage nach dessen Bedeutung und Umgang im menschlichen Leben verknüpft (Malpas und Lickiss 2012). Die Leidenslinderung stellt dabei einen wesentlichen Aspekt moralischen Handelns dar und wird von Joseph Amato als das Herzstück dessen beschrieben (Amato und Monge 1990). Der normative Charakter von Leiden liegt darin, dass sich eine Leidenserfahrung nicht nur auf den Leidenden selbst bezieht, sondern auch bei den Mitmenschen den Impuls auslöst, gegen dieses Leiden etwas tun zu wollen. Auch in reflexiver Hinsicht wird eine Leidenserfahrung bewertet und die Frage nach dem Sinn des eigenen Leidens gestellt (Bozzaro 2015c). 
Dabei ist diese Bewertung immer schon a priori kulturell determiniert, da die eigene Interpretation eines Leidenszustandes immer auch ein Produkt erlernter und sozialer Umgebungsfaktoren ist. Das Leiden nimmt gegebener Maßen die Bedeutung ein, die es im Kontext mit anderen Menschen und in dem Sinn des allgemein verstandenen Begriffes erlangt (Hoffmaster 2014).

Eine Form der Reaktion auf Leiden ist die Medizin, deren zentrales Handlungsmotiv die Leidenslinderung ist (Edwards 2003). In der Regel ist der Grund für eine Leidenslinderung die Behandlung von sowohl akuten und chronischen als auch benignen und malignen Krankheitsgeschehen. Nicht immer steht jedoch eine Krankheit, sondern vielmehr ein umfassendes Leiden des Patienten in einer speziellen Situation im Vordergrund einer Therapie, wie beispielsweise bei der Sterbebegleitung oder beim unerfüllten Kinderwunsch. Der Umgang mit diesen besonderen Fällen ist eine Herausforderung.

Der Leidensbegriff wurde im medizinethischen Kontext am Beispiel der palliativen Sedierung diskutiert. In diesem Zusammenhang hat sich gezeigt, dass Leiden als ein komplexes und heterogenes Geschehen möglicherweise nicht immer dem medizinischen Automatismus nach maximaler Leidenslinderung unterliegen sollte. Dies scheint einerseits gegenüber den verschiedenen Aspekten von Leiden nicht angemessen. Andererseits ist es bedenkenswert, ob jede zur Verfügung stehende Behandlungsmöglichkeit in den Dienst dieses Auftrages gestellt werden darf und sollte (Bozzaro 2015b). Der Absolutheitsanspruch des medizinischen Leidenslinderungsauftrages führt möglicherweise zu paradoxen und ethisch konfliktbeladenen Situationen. Eine vollkommene Leidenslinderung kann in manchen Fällen nur durch eine Abschaffung des Leidenden selbst erreicht werden (Bozzaro 2015b; Majumder 2014; Lauritzen 2014; Reichardt 2016; Green und Palpant 2014). Dies ist beim ärztlich assistierten Suizid der Fall (Bozzaro 2015b, 2015a). In Form eines ,vermeidbaren“ oder „unnötigen“ Leidens wird der Leidensbegriff beispielsweise auch als Argument zur Durchführung der pränatalen Diagnostik und Präimplantationsdiagnostik mit ihren Konsequenzen angeführt (Majumder 2014; Hepp 2006).

Die stetige Zunahme und Fülle von Behandlungsmöglichkeiten in der modernen Medizin wirft ein weiteres Problemfeld auf. Die Entgrenzung von Therapiemöglichkeiten lässt den Wunsch, dass jedes Leiden zu lindern ist, realisierbar erscheinen und führt zu hohen Machbarkeitserwartungen an die Medizin. Hierdurch wird Leiden zu etwas absolut Kontrollierbarem (Maio 2016). Im Zuge dieses Perspektivwechsels verändert sich folglich der Umgang mit dem Leiden sowie der Anspruch an dessen Linderung. Mit der Entwicklung neuer Behandlungsmöglichkeiten entsteht jedoch unter Umständen erst ein Bewusstsein, dass gewisse Zustände und Lebenssituationen als ein Leiden empfunden werden. Mit der Bestimmung, dass es Aufgabe der Medizin ist, Leiden zu lindern, wurde der Leidenslinderungsauftrag hauptsächlich in den medizinischen Sektor verschoben. Damit geht eine zunehmende Medikalisierung und Pathologisierung von körperlichen Zuständen einher, die lediglich eine Veränderung des Wohlbefindens darstellen. Jegliche Art der negativen Körperwahrnehmung führt zum Aufsuchen eines Arztes und wird damit als potentiell medizinisch behandlungswürdig eingestuft (Lauritzen 2014; Buyx und Hucklenbroich 2009). Die Schwierigkeit besteht eben darin, dem Leidenslinderungsauftrag ange- 
messen nachzukommen, ohne dass dabei das Leiden an sich als anthropologische Grundkonstante aus dem Blick gerät.

\section{Leidenskonzepte}

Aktuelle Leidenskonzeptionen bestimmen Leiden bezüglich der Person im Kontext ihres sinnstiftenden Lebensentwurfes. Eine Annäherung an den Leidensbegriff erfolgt somit nicht nach dem Leib-Seele-Dualismus, sondern ausgehend von dem Bedeutungshorizont der leidenden Person (Malpas 2016). Es lassen sich verschiedene Leidenskonzepte mit sowohl subjektivistischen als auch objektivistischen Grundpositionen sowie Mischpositionen unterscheiden. Gerade Letztere fokussieren sich nicht auf eine Festlegung hinsichtlich objektiver oder subjektiver Erkennbarkeit von Leiden und bieten damit einen anderen Ansatzpunkt zum Leidensverständnis.

Die wohl bekannteste Auseinandersetzung mit dem Leidensbegriff ist der subjektivistische Ansatz von Eric Cassell. Durch seine Grundannahme, dass in einem Leidenszustand die Integrität der Person in ihrer Gesamtheit zur Disposition steht, sind die ihm nachfolgenden Auseinandersetzungen mit dem Leidensbegriff maßgeblich beeinflusst worden. Leiden zu erfassen bedeutet demnach, die Person ganzheitlich, d.h. den leidenden Menschen im Kontext der gelebten Vergangenheit, zwischenmenschlicher Beziehungen, des jeweiligen kulturellen Hintergrundes inklusive spiritueller Bedeutungshorizonte und in Bezug auf die geplante Zukunft und den damit verbundenen Hoffnungen zu sehen und anzuerkennen, denn nur dann kann Leiden angemessen gelindert werden. Andernfalls ist die Entstehung neuen Leidens möglich (Cassel 1982; Cassell 2004). Cassell legt mit seinem Leidenskonzept einen nicht ganz unproblematischen subjektivistischen Leidensbegriff dar. Nur die Person, die das Leiden erfährt, kann ausdrücken, dass sie leidet. Somit hängt die Bewertung des eigenen Leidens vor allem von dem Bewusstsein ab, dass man leidet (Cassell 2014).

Sten van Hooft definiert Leiden im Gegensatz dazu im Rahmen eines objektivistischen Leidenskonzeptes. Angelehnt an die aristotelische Lehre wird Leiden als eine Verhinderung eines erfüllten Lebens bestimmt. Eine Person umfasst ihre biologische Funktion, ihre Emotionalität mit ihren Wünschen und Sehnsüchten, ihre Rationalität einschließlich der praktischen Lebensaspekte sowie die Sinn- und Bedeutungsgebung der eigenen Existenz. Können alle diese vier Aspekte im Leben eines Menschen gelebt werden, ist das Ziel menschlichen Lebens als ein erfülltes Leben möglich. Kommt es zur Verhinderung eines dieser Aspekte, liegt ein Leiden vor, das für Außenstehende ersichtlich sein sollte. Dabei muss dieses Leiden dem Leidenden nicht notwendigerweise selbst bewusst sein (van Hooft 1998). Die objektive Erkennbarkeit von Leiden stellt eine ebenso problematische Forderung dar, da diese unter anderem zu dem Paradoxon führt, dass objektiv ein Leiden vorliegt, aber der Leidende selbst sein Leiden nicht als Leiden wahrnimmt (Edwards 2003).

Eine weitere Bestimmung von Leiden stellt eine Erklärung über den Begriff der Lebensqualität dar. In diesem Zusammenhang bestimmt Barry Hoffmaster Leiden als ein Verlust von Werten, die das Leben zufrieden und bedeutungsvoll machen. Dies kann in verschieden qualitativen Abstufungen vorkommen: Die höchste Stufe 
von Leiden stellt der Verlust von lebenswerten Faktoren im Sinne eines maximalen Verlustes von Lebensqualität dar (Hoffmaster 2014). Verschiedene Untersuchungen konnten auch zeigen, dass in einem Leidenszustand die Lebensqualität des Einzelnen eingeschränkt ist (Land et al. 2012). Eine Leidenslinderung wiederum trägt unmittelbar zu einer Steigerung der Lebensqualität bei (Anderson 2013). Der Vorteil einer Begriffsbestimmung von Leiden anhand der Lebensqualität ist, dass die Festlegung eines Leidenszustandes erfolgt, ohne dass ein Bewusstsein von Leiden oder eine objektive Feststellbarkeit desselben als konstituierend für den Leidensbegriff angenommen werden muss.

Edwards distanziert sich ebenfalls von einer Festlegung hinsichtlich subjektivistischer oder objektivistischer Erkennbarkeit von Leiden und extrahiert nach einem linguistischen Vorgehen drei zentrale Merkmale von Leiden. Diese ist erstens die sogenannte phänomenologische Bedingung. Damit ist gemeint, dass Leiden etwas ist, was mittels des Empfindungsvermögens zunächst erstmal nur gefühlt werden kann. Zudem muss eine temporale Bedingung erfüllt sein, denn Leiden besteht über eine gewisse Zeit fort und ist zeitlich nicht limitiert. Als drittes Kriterium legt er fest, dass Leiden eine zentrale Stellung im Leben des Leidenden einnehmen muss. Leiden beeinträchtigt den individuellen Erlebniskontext (Edwards 2003).

Da Leiden als etwas Gefühltes angenommen wird, stellt sich allerdings die Frage, wie dieses Gefühlte letztendlich vermittelbar und für Außenstehende erkennbar wird. Zentraler Begriff hierbei könnte die Schwelle sein, oberhalb derer ein Betroffener seine Situation mit Leiden umschreiben würde. Eine Angabe über die Intensität des persönlichen Leidens in Form einer numerischen Skala erscheint, im Gegensatz zur Angabe über eine Schmerzintensität, unzureichend. Die Objektivierung von Schmerzen mittels einer Schmerzskala von eins bis zehn ist schon lange gute klinische Praxis und damit aussagekräftig. Ein Leiden lässt sich in gleicher Weise jedoch nicht standardisieren. Eine Objektivierung qualitativer Begrifflichkeiten stellt in der Wissenschaft und im klinischen Alltag weiterhin eine Herausforderung dar (Edwards 2003). Hinsichtlich des Verhältnisses des Leidensbegriffs zu dem Begriff Schmerzen, wurde ein synonymer Gebrauch bereits nach der cartesianischen Leib-Seele-Dichotomie, nach der Schmerz Ausdruck des Körpers und Leiden Ausdruck seelischer Vorgänge ist, abgelehnt. Aus Sicht der aufgeführten holistischen Leidenskonzeptionen werden Schmerzen vielmehr als eine Unterform von Leiden angesehen: Schmerzen können eine Ursache für Leiden sein, müssen aber nicht notwendig zu einem Leiden führen. Sie stellen demzufolge weder eine notwendige noch eine hinreichende Bedingung für ein Leiden dar (Hoffmaster 2014; Edwards 2003; Cassell 2004; van Hooft 1998).

\section{Der unerfüllte Kinderwunsch - eine Begriffsklärung}

In der Fortpflanzungsgeschichte des Menschen ist der unerfüllte Kinderwunsch als elementares menschliches Bedürfnis immer schon ein bedeutsames Problem. Dies zeigt uns beispielsweise die Geschichte von Sara und Abraham im Alten Testament der Bibel (Genesis 16 1-3. ELB 2006). In der heutigen Zeit bezeichnen wir den unerfüllten Kinderwunsch und die damit einhergehende ungewollte Kinderlo- 
sigkeit als Leiden an der Unfruchtbarkeit, dem potentiell mit einer medizintechnischen Intervention begegnet werden kann. Die WHO definiert einen unerfüllten Kinderwunsch als Sterilität, wenn es einem Paar nach einem Jahr regelmäßigen und ungeschützten Geschlechtsverkehr nicht gelingt, ein Kind zu zeugen (ZegersHochschild et al. 2009). Dabei ist der Anteil derjenigen Paare, die eine Kinderwunschtherapie in Anspruch nehmen, steigend (Czeromin et al. 2018). Als Ursache für eine Sterilität sind oft körperliche Funktionsstörungen, beispielsweise der Spermien oder der Eierstöcke, zu finden. Diese betreffen beide Geschlechter in gleicher Weise. In ca. 10-20\% lässt sich jedoch keine Ursache für den unerfüllten Kinderwunsch finden (Diedrich et al. 2013; Brugo-Olmedo et al. 2001). Der Stellenwert und der Einfluss psychischer Faktoren wie Depressivität, Stress und Ängstlichkeit auf die Fertilität wird jedoch widersprüchlich diskutiert. Wenn sowohl organische als auch psychische Ursachen für den unerfüllten Kinderwunsch ausgeschlossen wurden, liegt eine idiopathische Sterilität vor (Toth 2019). Es gilt zu beachten, dass der unerfüllte Kinderwunsch einen hohen Leidensdruck in Form einer psychischen und emotionalen Belastung erzeugt. Betroffene Paare, vor allem Frauen, nehmen einen unerfüllten Kinderwunsch als eine Lebenskrise wahr. Damit verbunden sind Ängstlichkeit, Versagensgefühle, depressive Verstimmungen, sozialer Rückzug und Schuldgefühle. Dies führt auch zu Konflikten in der Partnerschaft. Insgesamt ist die Lebensqualität dieser betroffenen Paare häufig eingeschränkt (Kentenich et al. 2014; Wischmann 2003; Stanhiser und Steiner 2018; Sexty et al. 2016). Das Leiden am unerfüllten Kinderwunsch stellt folglich eine Situation dar, in der durch die Verhinderung eines zutiefst menschlichen Bedürfnisses ein Leiden entsteht. Dieses Leiden enthält folglich eine psychoexistenzielle Dimension.

Aber auch Diagnostik und Therapie des unerfüllten Kinderwunsches sind seelisch und körperlich belastend. Das Aufsuchen eines Arztes erfolgt nicht auf Grund eines körperlichen Symptoms, sondern auf Grund einer Nichterfüllung eines Kinderwunsches im Rahmen einer Paarbeziehung. Eine Abklärung einer möglichen körperlichen Funktionsstörung, die zu einer Unfruchtbarkeit führt, betrifft demzufolge beide Partner. Damit bildet der unerfüllte Kinderwunsch den Ausgangspunkt für eine Reihe diagnostischer und operativer Verfahren sowie für die technischen Maßnahmen der assistierten Reproduktion zur Überwindung desselbigen. Diese Interventionen sind Eingriffe, welche insbesondere in die Integrität des Körpers der Frau unterschiedlich stark eingreifen und deshalb mit gewissen gesundheitlichen Risiken verbunden sind. Schon die Diagnostik ist häufig für die Kinderwunschpatientinnen mit einem operativen Eingriff in Form einer Bauchspiegelung verbunden. In Bezug auf den Fortgang der Kinderwunschbehandlung bestehen Risiken, welche durch die hormonelle Vorbehandlung und durch die Eizellentnahme bedingt sind, oder die aus der mittels assistierter Reproduktion entstandenen Schwangerschaft resultieren. Diese sind beispielsweise ein erhöhtes Risiko für einen Gestationsdiabetes, schwangerschaftsinduzierte Bluthochdruckerkrankungen oder für postpartale Blutungen (Cai et al. 2017; Nyfløt et al. 2017; Toth 2019). Ebenso gibt es bei einer ART das Risiko der Mehrlingsschwangerschaften, die wiederum mit einer erhöhten Rate an Komplikationen, wie beispielsweise die Frühgeburtlichkeit oder Präeklampsie einhergehen (Schröer und Weichert 2013). Auch für die mittels IVF und ICSI erzeugten Kinder werden im Vergleich zu Kindern nach Spontankonzeption soma- 
tische Risiken wie ein erhöhtes Krebs- und Fehlbildungsrisiko sowie ein erhöhtes Risiko für Hauterkrankungen beschrieben (Krieger et al. 2018; Wainstock et al. 2017; Davies et al. 2017). Die Kinderwunschbehandlung an sich kann mit einer psychischen Belastung im Sinne von Stress, Depressivität und Ängstlichkeit, die wiederum zu Partnerschaftskonflikten und sozialem Rückzug führen können, einhergehen (Kentenich et al. 2014). Das Leiden an einem unerfüllten Kinderwunsch stellt folglich die Voraussetzung zur Durchführung von Behandlungen dar, die mit einem gesundheitlichen Risiko für Körper und Psyche verbunden sind und deren Langzeitauswirkungen bisher nicht sicher geklärt sind.

\section{Der unerfüllte Kinderwunsch als Leiden}

Das Erleben einer ungewollten Kinderlosigkeit stellt einen Zustand dar, der in unterschiedlichen Ausprägungsformen als negative Erfahrung erlebt und bewertet werden kann. Dabei ist der unerfüllte Kinderwunsch meist nicht mit einem Schmerzerlebnis, sondern vielmehr mit der Erfahrung verbunden, dass existenzielle Zukunftsvorstellungen nicht eintreten. Es handelt sich somit um einen Umstand, der eng mit der persönlichen Lebensplanung und häufig ohne körperliche Symptome einhergeht. Die Konsultation eines Arztes erfolgt eben dann, wenn eine gewünschte Lebenssituation, nämlich die Gründung einer Familie, nicht eintritt. Wie die Ausführungen zum Leidensbegriff gezeigt haben, muss nicht notwendig ein organisch krankhafter Zustand vorliegen, um von einem Leiden zu sprechen. Gerade im Rahmen holistischer Leidenskonzepte wird den psychosozialen und sinngebenden Lebensaspekten mehr Bedeutung beigemessen und diese als ursächlich für ein Leiden angesehen.

Mehrfach ist die psychische Belastung des Leidens am unerfüllten Kinderwunsch beschrieben worden. Diese Situation wird von den betroffenen Paaren ähnlich einer Lebenskrise empfunden. Sie geht mit Wut, Trauer, Depression, sozialer Isolation, Schuldgefühlen und dem Gefühl des Kontrollverlustes einher. Dabei wird das Leiden gerade durch die Bedeutung, die dieser unerfüllte Wunsch in der Lebensplanung des Paares einnimmt, erzeugt (Hölzle und Wiesing 1991; Wischmann 2003; Strauß et al. 2004). Ein unerfüllter Kinderwunsch geht mit Einschränkungen der persönlichen Lebensqualität einher (Sexty et al. 2016). Betrachtet man das betroffene Paar im Rahmen seines Lebenskontextes und hinsichtlich seiner Lebensqualität, zeigt sich, dass der unerfüllte Kinderwunsch durchaus zu einem Leiden werden kann. Entsprechend der in der Darstellung der Leidenskonzeptionen besprochenen Leidensdeterminanten nach Edwards (die phänomenologische Bedingung (1), die temporale Bedingung (2) und die zentrale Stellung im Leben des Leidenden (3)), sollte die ungewollte Kinderlosigkeit ebenfalls als ein Leiden betrachtet werden: (1) Paare mit einem unerfüllten Kinderwunsch können ihre Situation als belastend und leidvoll wahrnehmen und beschreiben. (2) Das Ende des Zustandes der ungewollten Kinderlosigkeit ist sowohl bei regelmäßigem Geschlechtsverkehr als auch unter reproduktionsmedizinischen Maßnahmen nicht vorhersehbar. Diese Situation kann zusätzlich dadurch erschwert werden, dass als Folge des natürlichen Menstruationszyklusses der Frau regelmäßig Hoffnungen entstehen, die dann, bei nicht eingetretener Schwangerschaft, enttäuscht werden. Gefühle von Ohnmacht und Kon- 
trollverlust gehen damit einher und erzeugen in jedem neuen Zyklus eine diffuse Angst davor, dass es vielleicht nie klappen wird. Damit scheint gerade die temporale Bedingung beim unerfüllten Kinderwunsch ausschlaggebend und die Schwelle zu sein, oberhalb derer Paare ihre Situation als leidvoll ansehen. (3) Der Wunsch nach einer Familie betrifft die gesamte Lebensplanung eines Paares, da die Zukunftsplanung und die Vorstellungen von einer erfüllten Partnerschaft davon betroffen sind. Folglich nimmt der unerfüllte Kinderwunsch eine zentrale Stellung im Leben der Betroffenen ein.

Auch im Hinblick auf subjektivistische oder objektivistische Leidenskonzeptionen lässt sich der unerfüllte Kinderwunsch als ein Leiden beschreiben. Paare können zum einen ihre Situation der ungewollten Kinderlosigkeit als ein Leiden wahrnehmen und ausdrücken. Ihre Integrität als Person kann in dieser Situation durchaus zur Disposition stehen. Der Wunsch nach einer Familiengründung und das Nichteintreten dieser Situation hat in hohem Maße Auswirkungen auf den persönlichen Lebensplan des Paares, indem es die existenziellen persönlichen Lebensentwürfe betrifft. Zum anderen kann nach einer objektivistischen Leidenskonzeption der unerfüllte Kinderwunsch ebenso als ein Leiden angesehen werden. Familiengründung und Fortpflanzung mit der Schaffung neuer zwischenmenschlicher Beziehungen stellen einen wesentlichen Teil eines erfüllten menschlichen Lebens dar. Wird eine Verwirklichung dieses zentralen Lebensaspektes verhindert, kann ein Leiden vorliegen.

Entsprechend der aktuellen Leidenskonzepte sollte der unerfüllte Kinderwunsch als Leiden angesehen werden. Allerdings bleibt weiterhin zu klären, ob sich daraus unmittelbar eine Legitimierung für jegliche Form von reproduktionsmedizinischen Maßnahmen ableiten lässt.

Im Rahmen einer Auseinandersetzung von Sterilität als Krankheit wurde argumentiert, dass die Erfüllung eines Kinderwunsches nicht bloß als eine Erfüllung eines beliebigen Wunsches anzusehen ist, sondern einen normativen Bedürfnisanspruch darstellt. Demzufolge wird der Kinderwunsch nicht bloß als ein beliebiger Wunsch, sondern als ein elementares menschliches Bedürfnis mit normativer Implikation aufgefasst (Krones et al. 2006). Daraus lässt sich ableiten, dass der Kinderwunsch einen besonderen Stellenwert im Leben des Menschen einnimmt und eben nicht mit jedem beliebigen Wunsch gleichzusetzen ist. Die hier dargestellte Herleitung, den unerfüllten Kinderwunsch als Leiden anzunehmen, legt ebenfalls nahe, dass der Kinderwunsch ein elementares menschliches Bedürfnis darstellt. Dies liegt in der psychoexistenziellen Dimension von Leiden begründet. Wird der Kinderwunsch als ein normatives Bedürfnis aufgefasst, stellt dies durchaus eine Legitimation für somatische, reproduktive Interventionen dar. Fraglich bleibt aber, ob sich eine allumfassende Legitimation für jegliche Form reproduktiver Maßnahmen daraus ableiten lässt. Es bleibt dabei aber weiterhin fraglich, in welchem Ausmaß und für welche speziellen Fälle die Durchführung dieser medizinischen Interventionen gerechtfertigt ist. Die normativen Implikationen, die sich aus dem Leidensbegriff in diesem Kontext ergeben, bleiben somit klärungsbedürftig und werden im Folgenden diskutiert. 


\section{Der Leidenslinderungsauftrag der Medizin in der Reproduktionsmedizin}

In der medizinethischen Debatte ist zu beobachten, dass sich die Perspektive im Hinblick auf Leiden verändert hat. Es steht weniger die Frage nach dem „Wie“ einer Leidenslinderung im Vordergrund, sondern vielmehr beschäftigt und bestimmt die Frage, welches Leiden Gegenstand medizinischer Intervention sein sollte, den gegenwärtigen Diskurs (Callahan 2014). Diese Perspektivänderung in Bezug auf den Leidensbegriff ist vor dem Hintergrund der zunehmenden Fülle an Behandlungsmöglichkeiten und auf Grund der Medikalisierung von Leiden notwendig. Unter diesem Gesichtspunkt sollte auch der Leidenslinderungsauftrag der Medizin für den Bereich der Reproduktionsmedizin reflektiert und spezifiziert werden. Rechtfertigt das Leiden am unerfüllten Kinderwunsch eine Verwendung aller zur Verfügung stehenden technischen Möglichkeiten hinsichtlich der Leidenslinderung? Dies scheint unter zwei Gesichtspunkten nicht angemessen zu sein. Zum einen berührt das Leiden an einem unerfüllten Kinderwunsch das menschliche Selbstverständnis: Dies ist die Bedeutung von Leiden als anthropologischer Grundbedingung, verbunden mit dem elementaren Bedürfnis zur Fortpflanzung. Zum anderen bringen die somatischen Interventionen der ART gesundheitliche Risiken mit sich. Dabei müssen diese Therapien als Gesundheitsressourcen auch im Kontext der gegenwärtigen Herausforderungen einer angemessenen Allokation und deren Finanzierung gesehen werden (Zimmermann-Acklin 2011; Rauprich et al. 2011).

Das menschliche Leiden ist eine anthropologische Grundbedingung. Dies sollte in der Leidenslinderung nicht aus dem Blick geraten. Die Leidenslinderung des unerfüllten Kinderwunsches wird gerade dadurch komplex, dass es sich um die Linderung eines nicht in Erfüllung gehenden existenziellen Bedürfnisses handelt. Dahingehend konnte bereits gezeigt werden, dass der Kinderwunsch ein normatives Bedürfnis ist, das folglich einer Reaktion bedarf (Krones et al. 2006). Die Notwendigkeit, auf den unerfüllten Kinderwunsch in einer angemessenen Art und Weise zu reagieren, legt auch das hier dargestellte Ergebnis nahe. Eine Anwendung reproduktiver Techniken ohne strenge Indikationsstellung lässt sich daraus allerdings nicht unmittelbar ableiten. Dies hat folgende Gründe: Erstens hat sich gezeigt, dass der unerfüllte Kinderwunsch mit einer psychoexistenziellen Dimension einhergeht, die durch eine somatische Intervention nicht angemessen behandelt werden kann. Es ist also erforderlich, dass ein Schwerpunkt in der Behandlung auf diesem psychoexistentiellen Aspekt des Leidens liegt. Dabei muss beachtet werden, dass die Bewertung eines Leidenszustandes von subjektiven, kulturellen und gesellschaftlichen Faktoren abhängt. Hier sei auf das Argument der Authentizität von Leiden im Rahmen relationaler Autonomiekonzepte verwiesen, nach denen die Entscheidungen für medizinische Maßnahmen von dem gesellschaftlich Gewünschten beeinflusst werden kann (Bozzaro et al. 2019).

Zweitens kann im Rahmen einer Kinderwunschbehandlung das Leiden verstärkt werden. Die psychische Belastung dieser Therapie darf nicht unterschätzt werden und führt auch nicht selten zu einem Abbruch der Therapie. Es muss bedacht werden, dass eine einseitige Fokussierung auf die reproduktiven Interventionen zu einer Herabwürdigung von Paaren führen kann, die eine Therapie abbrechen oder sich ge- 
gen eine Therapie entscheiden. Eine Verabsolutierung der Leidenslinderung durch medizintechnische Intervention könnte auch dazu führen, dass Paare, die keine Therapie wünschen oder ein Kind adoptieren möchten, diskriminiert werden (McLeod 2017). Hinzu kommt, dass der unerfüllte Kinderwunsch auch als Folge einer veränderten Lebensplanung gesehen werden kann. Das Durchschnittsalter der Paare, die eine reproduktionsmedizinische Behandlung in Anspruch nehmen, ist in den letzten Jahren gestiegen (Czeromin et al. 2018). Dies ist unter anderem dadurch zu erklären, dass sich die Planung der eigenen Reproduktion verändert hat. Häufig wird die Familienplanung und Entscheidung zu einer Elternschaft auf Grund beruflicher Anforderungen oder eines fehlenden Partners ins höhere Alter verschoben. Aus der damit verbundenen verkürzten reproduktiven Phase folgt zusätzlich ein zunehmender Erfolgsdruck für das Eintreten einer Schwangerschaft (Strauß et al. 2004; Gnoth et al. 2003). In diesem Kontext ist es fraglich, ob das Leiden, welches durch eine bewusst herbeigeführte veränderte Lebensplanung entsteht, risikoreiche und teure Therapien rechtfertigt und ob eine Leidenslinderung unter diesem Aspekt überhaupt Gegenstand der Medizin sein sollte. In Bezug auf die veränderte Lebensplanung als Ursache für einen unerfüllten Kinderwunsch liegt es näher, die Vereinbarkeit von Familie und Beruf zu diskutierten und zu verbessern.

Drittens besteht auch immer die Möglichkeit, dass eine Kinderwunschbehandlung erfolglos bleibt. Die aktuellen Daten der assistierten reproduktionsmedizinischen Behandlung zeigen dies. Sowohl die Schwangerschaftsraten als auch die Geburtenraten variieren in Abhängigkeit von dem Alter der Frau, von den möglicherweise vorliegenden körperlichen Funktionsstörungen sowie von der eingesetzten Technik. Bezogen auf das ganze Kollektiv liegt die Baby-take-home-Rate bei ca. $20 \%$ (Czeromin et al. 2018). Daraus wird zum einen deutlich, dass ein nicht geringer Anteil der Paare ohne Erfolg aus einer Kinderwunschbehandlung geht und dauerhaft kinderlos bleibt. Deshalb sollte bedacht werden, dass der Versuch einer Leidenslinderung, auch durch eine ausschließlich medizintechnische Intervention, kein Garant zur Linderung darstellt. Auch Paaren, bei denen eine Therapie erfolglos verläuft, sollten Beratung und Unterstützung angeboten werden, um die Situation des unerfüllten Kinderwunsches in ihren Lebenskontext zu integrieren.

Die variierenden und individuellen Erfolgsaussichten einer Kinderwunschbehandlung sowie die mit einer Kinderwunschbehandlung einhergehenden gesundheitlichen Risiken für Mutter und Kind lassen eine Rechtfertigung der medizinischen Interventionen durch das Leiden am unerfüllten Kinderwunsch ebenfalls nicht unproblematisch erscheinen. Nicht nur die Kinderwunschbehandlung an sich geht mit gesundheitlichen Risiken einher, sondern auch die nach einer reproduktionsmedizinischen Behandlung entstandenen Schwangerschaften sind mit einer erhöhten Rate an Komplikationen verbunden. Die Langzeitfolgen dieser Therapien für Mutter und Kind sind bisher nicht endgültig geklärt (Toth 2019; Wenderlein 2018). Ein weiterer Aspekt, der bei der Beantwortung der Frage nach der Legitimierung der somatischen Interventionen zur Erfüllung einer ungewollten Kinderlosigkeit mit bedacht werden sollte, ist die Finanzierung der Therapien. Im Rahmen der Verknappung der Finanzmittel für Gesundheitsgüter ist zu beachten, dass die reproduktiven Maßnahmen kostenintensiv sind und eine Finanzierung sowohl über Selbstzahlung als 
auch durch Übernahme der Kosten durch die Krankenkassen zu diversen ethischen Problemen der Verteilungsgerechtigkeit und Fairness führen.

Es sollte anerkannt werden, dass ein unerfüllter Kinderwunsch ein Leiden ist. Allerdings ist es geboten, im Einzelfall differenziert zu prüfen, ob eine somatische Intervention sinnvoll erscheint. Eine Behandlung ist nur im Einzelfall und nach einer strengen Indikationsstellung zu rechtfertigen. Die kürzlich im deutschsprachigen Raum erstmals erschienene Leitlinie mit dem Titel „Diagnostik und Therapie vor einer assistiert reproduktionsmedizinischen Behandlung" hebt diese Wichtigkeit einer differenzierten Diagnostik vor einer ART hervor. Sie stellt eine fachübergreifende Handlungsanweisung dar, um die Schritte der Diagnostik bei bestehender Kinderlosigkeit zu standardisieren (Toth 2019).

Eine ausführliche Diagnostik und Beratung über die Auswirkungen und individuellen Erfolgsaussichten einer Kinderwunschbehandlung sind demnach erforderlich, um dem Leiden eines unerfüllten Kinderwunsches von Anfang an angemessen zu begegnen. Dabei ist im Rahmen einer individuellen und ergebnisoffenen Beratung inklusive psychosomatischer Mitbetreuung zu klären, ob eine medizinische Intervention mit all ihren Risiken eine angemessene Behandlung darstellt. In Form von zunehmenden Forderungen nach einer psychosomatischen Betreuung wird bereits versucht, die psychosozialen Aspekte des unerfüllten Kinderwunsches und seiner Behandlung zu berücksichtigen (Toth 2019; Stanhiser und Steiner 2018; StöbelRichter et al. 2013). Diese psychosoziale Begleitung der Paare ist bereits vor einer Behandlung und auch nach einer Behandlung bedeutsam, wie auch die Ergebnisse dieses Aufsatzes nahelegen. Allerdings wird dies, obwohl schon seit längerem gefordert, bisher nicht zufriedenstellend umgesetzt (Stöbel-Richter et al. 2011; Dorn und Wischmann 2013). Der Leidenslinderungsauftrag der Medizin sollte demzufolge für den Bereich der ART nicht eindimensional als eine Leidenslinderung durch eine ausschließlich somatisch fokussierte Therapie verstanden werden, sondern muss auf die psychoexistenzielle Dimension von Leiden abzielen.

\section{Schlussfolgerung}

Durch die Analyse der gängigen Leidenskonzeptionen konnte gezeigt werden, dass der unerfüllte Kinderwunsch die Kriterien von Leiden erfüllt. Damit kommt dem unerfüllten Kinderwunsch ein normativer Stellenwert zu, dessen Operationalisierbarkeit im Hinblick auf den Leidenslinderungsauftrag der Medizin problematisch ist. In diesem Zusammenhang hat sich gezeigt, dass der Absolutheitsanspruch an Leidenslinderung differenziert betrachtet werden muss und dabei nicht vergessen werden darf, dass Leiden eine „conditio humana“ darstellt. Dies ist vor dem Hintergrund der Fülle an Behandlungsmöglichkeiten sowie den stetig zunehmenden technischen Errungenschaften und den damit einhergehenden Machbarkeitserwartungen an die moderne Medizin erforderlich.

Eine ausschließlich auf eine medizinische Intervention fokussierte Kinderwunschbehandlung verfehlt den Anspruch auf Leidenslinderung, da hierbei wesentliche Faktoren nicht berücksichtigt werden. Beim Leiden an einem unerfüllten Kinderwunsch sind erstens die psychosozialen und existenziellen Vorstellungen des Lebens 
von besonderer Bedeutung und bedürfen einer Resonanz. Zweitens kann Leiden im Rahmen einer Kinderwunschbehandlung erst entstehen oder sich verstärken. Drittens gilt es zu beachten, dass ein nicht geringer Anteil der Paare auch nach einer Kinderwunschbehandlung dauerhaft kinderlos bleibt. Deshalb sind eine sorgfältige Diagnostik und strenge Indikationsstellung zur somatischen Intervention mit einer individuellen und ergebnisoffenen Beratung über die Risiken und Erfolgsaussichten geboten. Ebenso sind Unterstützungsangebote hinsichtlich der psychischen Belastungen, die mit einem unerfüllten Kinderwunsch einhergehen, notwendig. Vor diesem Hintergrund wäre es wünschenswert, die Therapie des unerfüllten Kinderwunsches hinsichtlich der „Wunscherfüllenden Medizin“ zu diskutieren.

Im Kontext der dargestellten Forderungen sollte immer bedacht werden, dass jedwedes medizinische Handeln, das der Verringerung von Leiden dient, schließlich in einen gesundheitspolitischen Kontext gebracht werden muss, in dem auch Fragen zur Sinnhaftigkeit, Priorisierung und Allokation beantwortet werden müssen.

\section{Einhaltung ethischer Richtlinien}

Interessenkonflikt A.M. Westermann und I. Alkatout geben an, dass kein Interessenkonflikt besteht.

Ethische Standards Für diesen Beitrag wurden von den Autoren keine Studien an Menschen oder Tieren durchgeführt. Für die aufgeführten Studien gelten die jeweils dort angegebenen ethischen Richtlinien.

Open Access Dieser Artikel wird unter der Creative Commons Namensnennung 4.0 International Lizenz (http://creativecommons.org/licenses/by/4.0/deed.de) veröffentlicht, welche die Nutzung, Vervielfältigung, Bearbeitung, Verbreitung und Wiedergabe in jeglichem Medium und Format erlaubt, sofern Sie den/die ursprünglichen Autor(en) und die Quelle ordnungsgemäß nennen, einen Link zur Creative Commons Lizenz beifügen und angeben, ob Änderungen vorgenommen wurden.

\section{Literatur}

Amato JA, Monge D (1990) Victims and values. A history and a theory of suffering. Praeger, New York Anderson RE (2013) Human suffering and quality of life. Conceptualizing stories and statistics. Springer, Heidelberg

Bozzaro C (2015a) Ärztlich assistierter Suizid: Kann „,unerträgliches Leiden“ ein Kriterium sein? Dtsch Med Wochenschr 140(02):131-134

Bozzaro C (2015b) Der Leidensbegriff im medizinischen Kontext: Ein Problemaufriss am Beispiel der tiefen palliativen Sedierung am Lebensende. Ethik Med 27(2):93-106

Bozzaro C (2015c) Schmerz und Leiden als anthropologische Grundkonstanten und als normative Konzepte in der Medizin. In: Maio G, Bozzaro C, Eichinger T (Hrsg) Leid und Schmerz. Konzeptionelle Annäherungen und medizinethische Implikationen. Karl Alber, Freiburg München, S 13-36

Bozzaro C, Krause F, Weismann M (2019) Uterustransplantation. Ethisch gerechtfertigt? Ethik Med 31(2):113-129

Brugo-Olmedo S, Chillik C, Kopelman S (2001) Definition and causes of infertility. Reprod Biomed 2(1):173-185

Buyx A, Hucklenbroich P (2009) „Wunscherfüllende Medizin“ und Krankheitsbegriff: Eine medizintheoretische Analyse. In: Kettner M (Hrsg) Wunscherfüllende Medizin - Ärztliche Behandlung im Dienst von Selbstverwirklichung und Lebensplanung. Campus, Frankfurt, S 25-53

Cai S, Natarajan P, Chan J, Wong PC, Tan K, Godfrey K, Gluckman P, Shek L, Yap F, Kramer M (2017) Maternal hyperglycemia in singleton pregnancies conceived by IVF may be modified by first-trimester BMI. Hum Reprod 32(9):1941-1947

Callahan D (1999) Remembering the goals of medicine. J Eval Clin Pract 5(2):103-106

Callahan D (2014) Foreword. In: Green RM, Palpant NJ (Hrsg) Suffering and bioethics. Oxford University Press, Oxford, S ix-xi

Cassel EJ (1982) The nature of suffering and the goals of medicine. N Engl J Med 306(11):639-645 
Cassell EJ (2004) The nature of suffering and the goals of medicine. Oxford University Press, Oxford

Cassell EJ (2014) Suffering and human dignity. In: Green RM, Palpant NJ (Hrsg) Suffering and bioethics. Oxford University Press, Oxford, S 15-30

Czeromin U, Krüssel J, Tandler-Schneider A (2018) Jahrbuch 2017 - D.I.R. - Deutsches IVF Register. J Reproduktionsmed Endokrinol 15(5/6):1-55

Davies M, Rumbold A, Marino J, Willson K, Giles L, Whitrow M, Scheil W, Moran L, Thompson J, Lane M (2017) Maternal factors and the risk of birth defects after IVF and ICSI: a whole of population cohort study. BJOG 124(10):1537-1544

Diedrich K, Ludwig M, Griesinger G (2013) Reproduktionsmedizin. Springer, Heidelberg

Dorn A, Wischmann T (2013) Psychologische Aspekte der Reproduktionsmedizin. Gynäkologe 46(12): 913-917

Edwards SD (2003) Three concepts of suffering. Med Health Care Philos 6(1):59-66

Genesis 16 (2006) 1-3. ELB. In: Die Bibel nach der Elberfelder Übersetzung, R. Brockhaus Verlag Wuppertal, Christliche Verlagsgesellschaft Dillenburg, Bd 1

Gnoth C, Frank-Herrmann P, Freundl G (2003) Angepasstes Management bei ,unerfülltem Kinderwunsch“. Geburtshilfe Frauenheilkd 63(02):124-129

Green RM, Palpant NJ (2014) Suffering and bioethics. Oxford University Press, Oxford

Hepp H (2006) Pränataldiagnostik. Gynäkologe 39(11):861-869

Hoffmaster B (2014) Understanding suffering. In: Green RM, Palpant NJ (Hrsg) Suffering and bioethics. Oxford University Press, Oxford, S 31-53

Hölzle C, Wiesing U (1991) In-Vitro Fertilisation: Ein umstrittenes Experiment: Fakten, Leiden, Diagnosen, Ethik. Springer, Berlin

van Hooft S (1998) Suffering and the goals of medicine. Med Health Care Philos 1(2):125-131

Jaspers K (1973) Philosophie II Existenzerhellung. Springer, Berlin

Kentenich H, Brähler E, Kowalcek I, Strauß B, Thorn P, Weblus AJ, Wischmann T, Stöbel-Richter Y (2014) Leitlinie psychosomatisch orientierte Diagnostik und Therapie bei Fertilitätsstörungen. PsychosozialVerlag, Gießen

Kettner M (2006) Assistenz zum guten Leben. Ethik Med 18(1):5-9

Kettner M (2009) Wunscherfüllende Medizin - Ärztliche Behandlung im Dienst von Selbstverwirklichung und Lebensplanung. Campus, Frankfurt

Krieger Y, Wainstock T, Sheiner E, Harlev A, Landau D, Horev A, Bogdanov-Berezovsky A, Walfisch A (2018) Long-term pediatric skin eruption-related hospitalizations in offspring conceived via fertility treatment. Int J Dermatol 57(3):317-323

Krones T, Neuwohner E, El Ansari S, Wissner T, Richter G (2006) Kinderwunsch und Wunschkinder. Ethik Med 18(1):51-62

Land KC, Michalos AC, Sirgy MJ (2012) Prologue: The development and evolution of research on social indicators and quality of life (QOL). In: Land KC, Michalos AC, Sirgy MJ (Hrsg) Handbook of social indicators and quality of life research. Springer, Dordrecht, S 1-22

Lauritzen P (2014) Reproductive technology in suffering's shadow. In: Green RM, Palpant NJ (Hrsg) Suffering and bioethics. Oxford University Press, Oxford, S 357-373

Maio G (2016) Der Schmerz als Widerfahrnis. Die Kontrollierbarkeitserwartung als Problem. In: Maio G, Bozzaro C, Eichinger T (Hrsg) Leid und Schmerz. Konzeptionelle Annäherungen und medizinethische Implikationen. Karl Alber, Freiburg, München, S 169-179

Majumder MA (2014) Preimplantation genetic diagnosis and the prevention of suffering. In: Green RM, Palpant NJ (Hrsg) Suffering and bioethics. Oxford University Press, Oxford, S 404-427

Malpas J (2016) Suffering. In: Ten Have H (Hrsg) Encyclopedia of global bioethics. Springer, Heidelberg, S $2733-2742$

Malpas J, Lickiss N (2012) Perspectives on human suffering. Springer, Heidelberg

McLeod C (2017) The medical nonnecessity of in vitro fertilization. Int J Fem Approaches Bioeth 10(1):78-102

Montgomery F, Nieschlag E, Krüssel J, Hepp H (2018) Richtline zur Entnahme und Übertragung von menschlichen Keimzellen im Rahmen der assistierten Reproduktion. Dtsch Arztebl 115(22):A1096

Nyfløt L, Sandven I, Oldereid N, Stray-Pedersen B, Vangen S (2017) Assisted reproductive technology and severe postpartum haemorrhage: a case-control study. BJOG 124(8):1198-1205

Rauprich O, Berns E, Vollmann J (2011) Die Finanzierung der Reproduktionsmedizin. Gynäkol Endokrinol 9(1):60-68

Reichardt J-O (2016) Zumutbares Leiden: Ausmaße und Grenzen in der Medizin. In: Maio G, Bozzaro C, Eichinger T (Hrsg) Leid und Schmerz. Konzeptionelle Annäherungen und medizinethische Implikationen. Karl Alber, Freiburg München, S 202-231 
Schröer A, Weichert J (2013) Mehrlingsschwangerschaften. In: Diedrich K, Ludwig M, Griesinger G (Hrsg) Reproduktionsmedizin. Springer, Heidelberg, S 329-337

Sexty R, Griesinger G, Kayser J, Lallinger M, Rösner S, Strowitzki T, Toth B, Wischmann T (2016) Fertilitätsbezogene Lebensqualität bei Patientinnen in deutschen Kinderwunschzentren. Geburtshilfe Frauenheilkd 76(02):V14

Stanhiser J, Steiner AZ (2018) Psychosocial aspects of fertility and assisted reproductive technology. Obstet Gynecol Clin 45(3):563-574

Stöbel-Richter Y, Sender A, Brähler E, Strauß B (2013) Psychologische Beratung und Psychotherapie bei ungewollter Kinderlosigkeit. Psychother Psychosom Med Psychol 63(09/10):400-414

Stöbel-Richter Y, Thorn P, Brähler E, Kentenich H, Wischmann T (2011) Umfrageergebnisse zum Stellenwert psychosozialer Beratung in reproduktionsmedizinischen Zentren in Deutschland - eine Pilotstudie. J Reprodmed Endokrinol 8(6):416-423

Strauß B, Henning K, Beyer K, Hoppe I, Starker W (2004) Ungewollte Kinderlosigkeit. Gesundheitsberichterstattung des Bundes, Bd. 20. Robert Koch-Institut, Berlin

Toth B (2019) Diagnostik und Therapie vor einer assistierten reproduktionsmedizinischen Behandlung (ART). S2k-Leitlinie, AWMF-Register (015/085):015-085

Wainstock T, Walfisch A, Shoham-Vardi I, Segal I, Harlev A, Sergienko R, Landau D, Sheiner E (2017) Fertility treatments and pediatric neoplasms of the offspring: results of a population-based cohort with a median follow-up of 10 years. Am J Obstet Gynecol 216(3):314.e1-314.e14

Wehmeyer E (2012) Kostenübernahme bei künstlicher Befruchtung. Gynäkologe 45(6):487-491

Wenderlein JM (2018) IVF-Kinder langfristig nachbeobachten? Frauenarzt 59(10):794-798

Wischmann T (2003) Psychosoziale Aspekte bei Fertilitätsstörungen. Gynäkologe 36(2):125-136

Zegers-Hochschild F, Adamson GD, de Mouzon J, Ishihara O, Mansour R, Nygren K, Sullivan E, Van der Poel S (2009) The international committee for monitoring assisted reproductive technology (ICMART) and the world health organization (WHO) revised glossary on ART terminology, 2009. Hum Reprod 24(11):2683-2687

Zimmermann-Acklin M (2011) Wie können faire Allokationsentscheidungen auf der Makroebene getroffen werden? Bioeth Forum 4(3):81-87 